\title{
Infrared thermography-based integrated approach aimed at objective evaluation of systemic vascular reactivity in humans
}

\author{
by B. G. Vainer* and V. V. Morozov**
}

\author{
* Rzhanov Institute of Semiconductor Physics SB RAS, Novosibirsk State University; 13 Lavrentyev av., \\ Novosibirsk, 630090, Russia, BGV@isp.nsc.ru \\ ** Institute of Chemical Biology and Fundamental Medicine SB RAS; 8 Lavrentyev av., \\ Novosibirsk, 630090, Russia, doctor.morozov@mail.ru
}

\section{Standard thermal imaging-based diagnostics as applied to neurology}

Thermal imaging has long been in use as a prospective diagnostic method in medicine. Infrared cameras are often involved in the diagnostic process to pick up valuable information about blood flow intensity in the peripheral vessels. Infrared thermography-based data allow receiving additional information about the state of microcirculation in tissues in various pathological cases. In practice, the method is applicable to many areas of medicine by virtue of its noninvasiveness and lack of contraindications. In particular, this is especially true to neurology and acute cerebral circulatory disorders. Diagnostic data obtained with the use of thermal imaging allow the physician to examine a functioning of the circulatory system in details and individualize for each patient a personalized treatment program for his rehabilitation after stroke. Infrared thermography, as applied to medicine, is capable of bringing invaluable information about human organism, and this information cannot be performed using any different diagnostic methods. We confirmed this fact for the umpteenth time by the ordinary study of patients suffered by typical aftereffects of the stroke.

The well-known dangerous outcomes of a stroke are paralysis and paresis of the extremities. In these cases, a comprehensive standard rehabilitation program does not always lead to improvement. We have developed and tested the non-standard method of complex examination of patients affected by ischemic stroke. The method is based on the simultaneous use of the FPA-based thermal imaging and some additional medical diagnostic means. Our main aim in this work was to assess objectively the results of treatment and rehabilitation of a patient affected by stroke on the base of examination of his microcirculation status in the affected limbs. An integrated approach to the assessment of microcirculatory disorders during rehabilitation after stroke provides an opportunity for a short-time (15-20 minutes) intensive investigation in order to identify the true physiological status of the patient's organism, to determine the level of vascular reactivity and give an objective prognosis on the disease in question.

Using the created diagnostic complex we examined 69 patients. The remoteness of their stroke coming varied from 1 month to 5 years with the rehabilitation course term up to 1 month and multiplicity of courses - 1-4 per year. After rehabilitation courses, positive dynamics in the neurological status of the patients undergone medical examination using a standard routine procedure occurred in $94 \%$ of individuals -64 persons (figure 1a). Improvement of microcirculation in the paretic limbs, according to infrared plethysmography (figure $1 \mathrm{~b}$ ), was registered in $75 \%$ (52 patients). Positive dynamics according to laser flowmetry (figure 1c) in the form of improved microcirculation and vessel filling was observed in $70 \%$ (50 patients). According to electroneuromyography (in the form of a rise of M-wave) the improvement of neuroreflex conductivity (figure 1d) in the muscles of paretic limb was registered in 46 patients $(67 \%)$. At the same time, according to the thermal imaging study (figure 1e), amelioration in the blood flow dynamics in the paretic limbs took place just in $58 \%$ of cases (40 patients). Determination of thermographic dynamics was carried out quantitatively, based on the analysis of two-dimensional thermograms. Typical thermograms exhibiting the affected lower limbs of stroke patients are given in figure 2. These examples do not give a chance to doubt in the validity and reliability of infrared thermographybased diagnostic results, as applied to the disorders in question.

\section{Integrated approach to thermal imaging-based medical diagnostics}

Recent researches have proved that modern computerized FPA-based infrared cameras make it possible in medicine and physiology to implement an up-to-date method called interventional infrared thermal diagnostics [1]. It provides an opportunity to increase the reliability of the results obtained from infrared thermography (IRT) examination in humans. At the same time, IRT alone is quite often inadequate to give exhaustive information about the current state of cardiovascular system. An alternative hardware environment needs to be involved into diagnostic process. The support of interventional infrared thermal examination by other medical control devices helps to advance the degree of diagnosis accuracy [2]. In this contribution, the structure of such an autonomous clinical thermal imaging-based diagnostic complex is described and some results obtained with its help devoted to vascular reactivity in humans are presented.

In addition to FPA-based infrared camera TKVr-IFP served as a main diagnostic instrument, several complementary techniques and methods were included in the complex. Among them were electrocardiography, laser Doppler flowmetry (LDF), pulse oximetry, sphygmography and noninvasive blood pressure determination. As a rule, all above-mentioned facilities were used simultaneously.

Similar to that shown in [1], a radically different systemic vascular reaction to short-term local occlusion in one of 


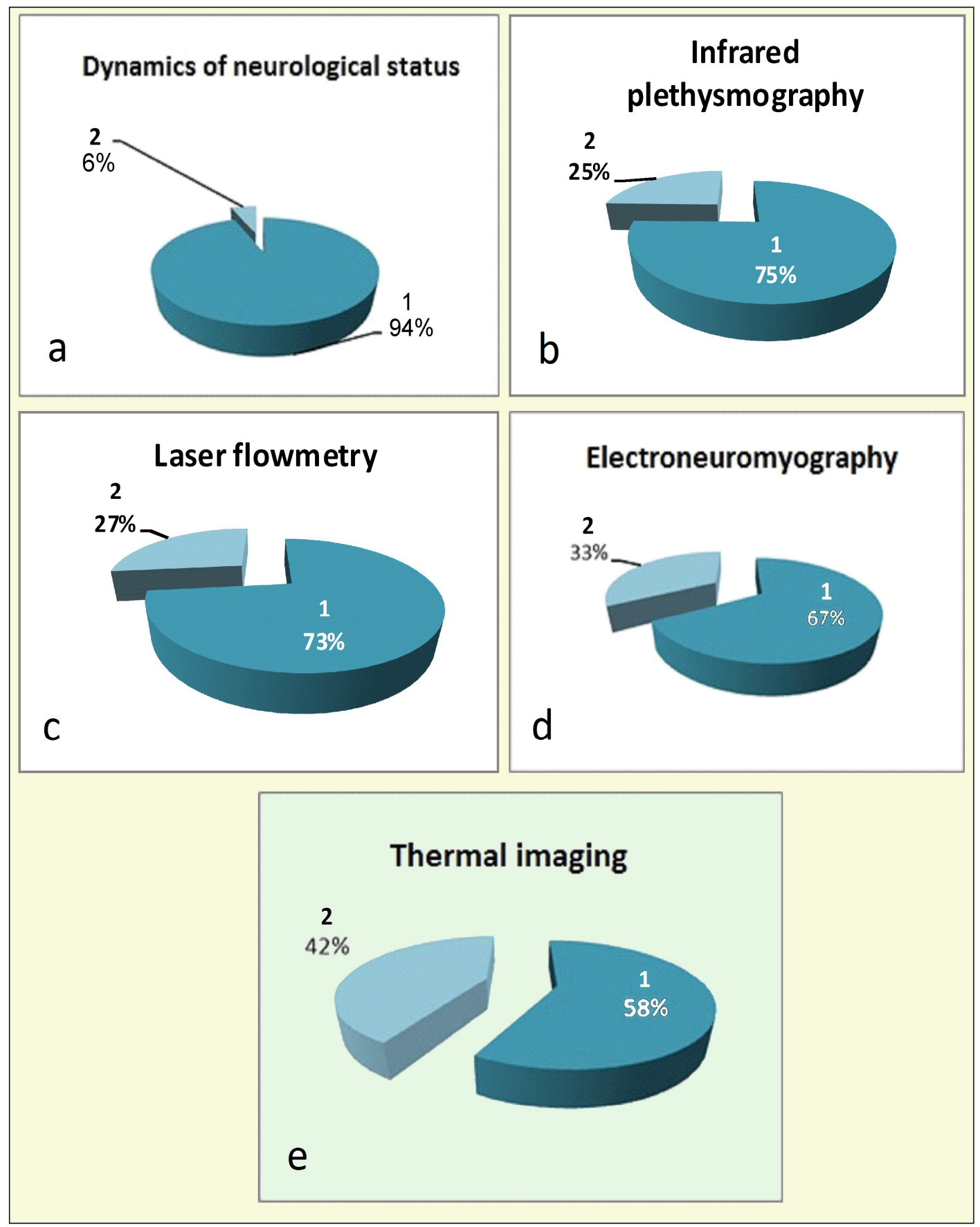

Fig. 1. Diagrams evaluating the sensitivity of different diagnostics methods to residual aftereffects of stroke.

the limbs - from full synchronism to complete indifference - was recorded among the examined individuals. Supporting examples are given in figure 3 . Figure 4 shows the results of diagnostic measurements obtained by LDF synchronously with the thermal imaging. Similarly to IRT data, LDF characteristics demonstrate the response to interventional impact, although it is less clear than that obtained with thermal imaging. Nevertheless, it is seen that the behavior of LDF-gram of the indifferent patient is distinct from that of the labile one.

Figure 5 represents the results obtained in the labile patient by means of pulse oximetry simultaneously with thermal imaging and LDF. We can see a slight reaction in contralateral hand to interventional impact: the content of oxygen is increased by about 1 percent, and the average heart rate also slightly rises. 


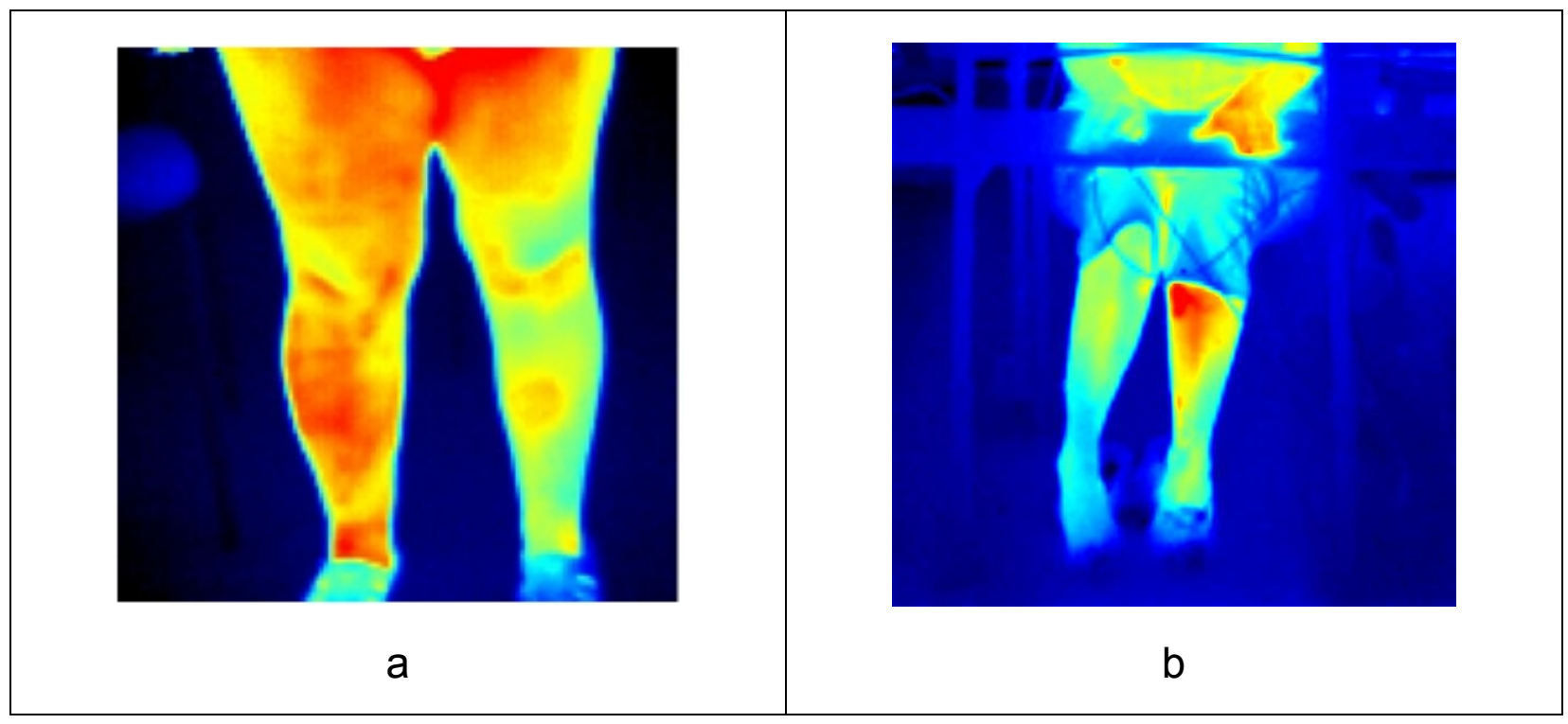

Fig. 2. Marked reduction in blood flow in the vessels of the lower extremities; after stroke condition. Paresis: $a$ - on the left side of body, $b$ - on the right side of body.

\section{Hypo- and hyperoxia-induced thermal manifestations}

We also attempted to develop the IRT-assisted method of vascular reactivity determination based on the observation of systemic response of the organism to general hypoxia and/or hyperoxia. These conditions appear in the course of routine surgical procedures under general anesthesia with the implementation of artificial pulmonary ventilation at the stage of initial narcosis. The examples of temperature change in the fingers of the right hand in the course of a standard stage of initial narcosis and trachea intubation are shown in figures 6 and 7 . The increased oxygen content in the gas mixture causes a sharp temperature rise in fingers and further - in the whole hand.

The expected temperature drop at the stage of hypoxia is not observed in this example, as the initial temperature of the fingers was comparable to the temperature of the ambient and heat exchange mechanisms that could cause further cooling of the skin missed.

\section{Conclusions}

The obtained results reaffirm a high sensitivity of the FPA-based thermal imaging in the assessment of blood supply in the affected organism limbs. Application of this method to neurology helps to optimize the course of rehabilitation and gives an instrument to control the efficacy of treatment. Using diagnostic protocol assessment of autonomic nervous system disorders after stroke, it is possible to increase the rehabilitation efficiency due to individualization of rehabilitation program based on identified microcirculatory disorders.

FPA-based infrared thermography may serve as valuable method allowing a precise detection of violations in the central regulation of microcirculation in limbs after stroke. Asymmetric patterns in thermal representation of the body indicated on two-dimensional thermograms allow judging about the functioning of the autonomic nervous system.

The integrated approach to the studies of systemic cardiovascular reactivity in patients and normal humans may be realized by a synchronous use of several diagnostic methods: IRT, LDF and pulse oximetry. This approach may be called "supplemented IRT". IRT-based criterion of systemic cardiovascular reactivity may serve as valuable index in emergency medicine, as it was explained in [3]. No. 40-B-2012).

This research was supported by the Siberian Branch of the Russian Academy of Sciences (Integrative Project

\section{REFERENCES}

[1] Vainer B. G., "Interventional infrared thermal diagnostics in medicine and physiology", E-Book Proceedings of 11th International Conference on Quantitative InfraRed Thermography, QIRT 2012, 11-14 June, 2012, NaplesItaly. Italy: University of Naples, paper ID-340, 10 pp., 2012.

[2] Vainer B. G., Morozov V. V., "Modern trends in medical infrared thermography", Proceedings of the Scientific Conference Application of Fundamental Sciences to Medicine, 2012, Novosibirsk, Russia, p. 20, 2012. (in Russian) 
[3] Vainer B. G., Markel A. L. "Imaging and quantitative characterization of bilateral vasomotor reactions in humans using high-performance thermography", QIRT10: Proceedings of the 10th edition of the Quantitative Infrared Thermography/ International Conference. July 27-30, 2010, Quebec City (Canada). X.P.V. Maldague, Editor. Canada: Universite Laval, pp. 91-94, 2010.

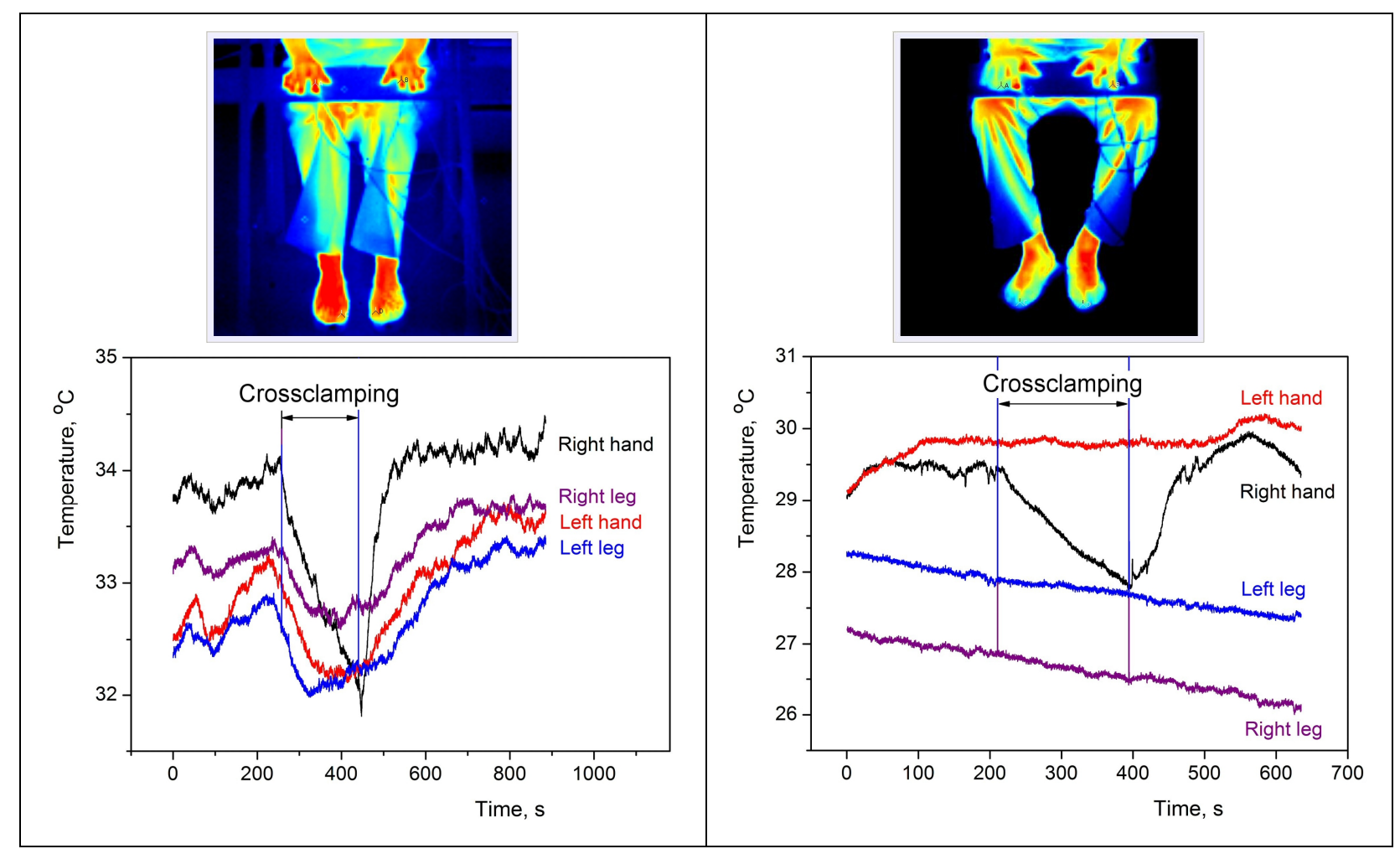

Fig. 3. Temperature variation in fingers and toes before, during and after a short (3 minutes) compressive occlusion of the right hand great vessels. Left: individual, who is sensitive to the interventional impact (labile patient); right: an example of an indifferent patient.

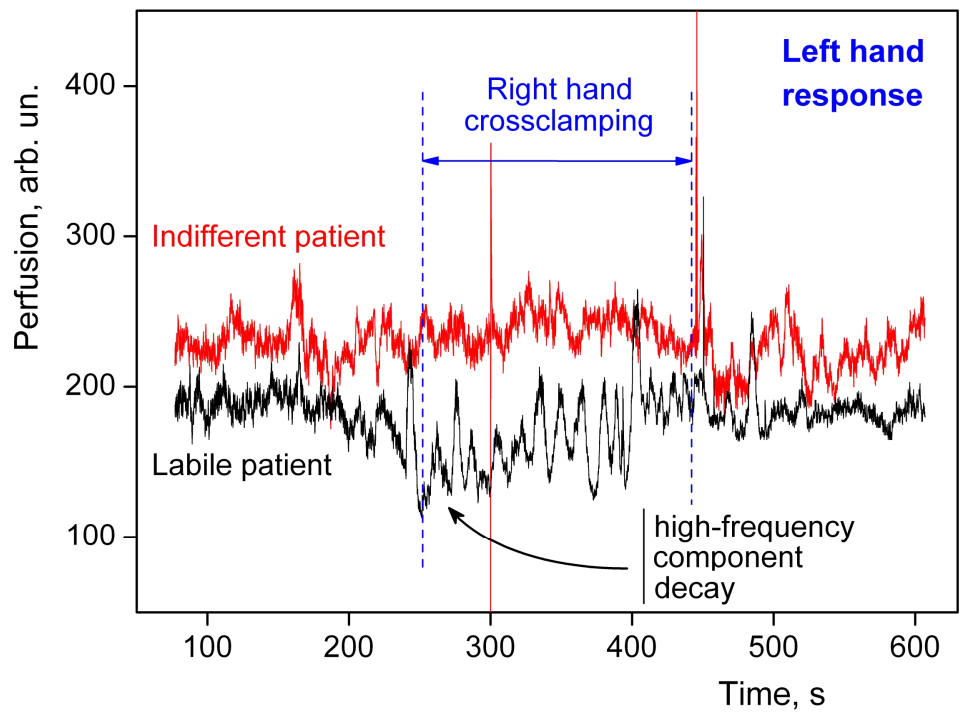

Fig. 4. Comparison of LDF responses of the left hand fingers before, during, and after a short (3 minutes) compressive occlusion of great vessels of the contralateral hand. The data were obtained in two individuals with different systemic vascular reactivities exhibited in figure 3. 


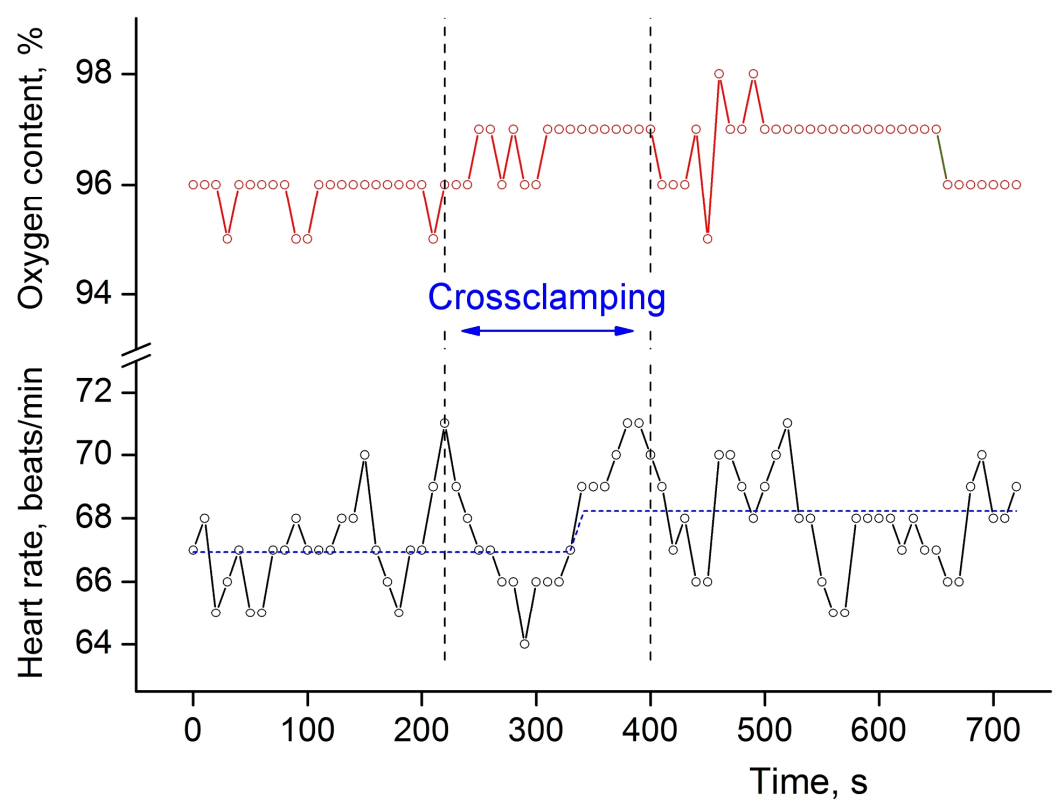

Fig. 5. Heart rate and the percentage of blood oxygen content before, during, and after a short (3 minutes) crossclamping of great vessels of the labile patient's hand. The characteristics were registered using the Patient Monitor GoldWay UT 4000 A, Gold Way Inc., China.

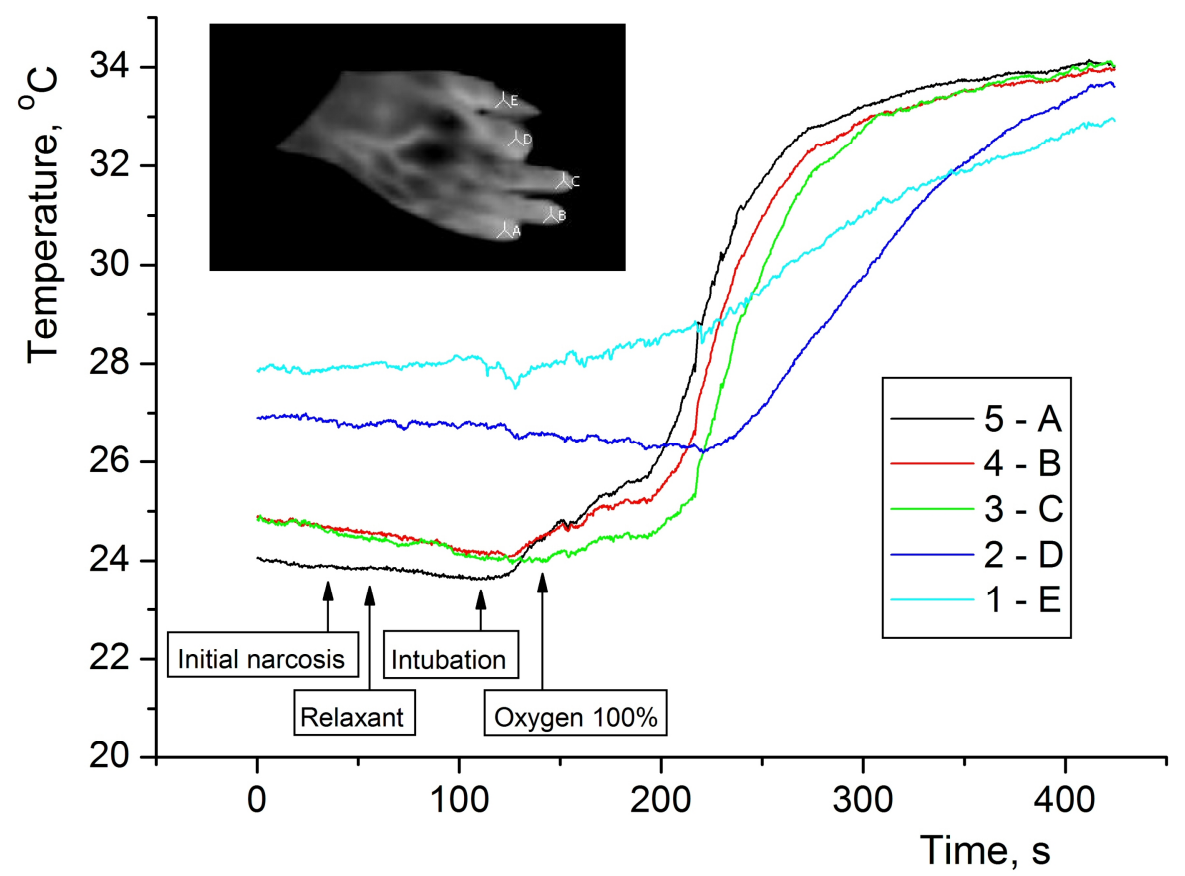

Fig. 6. Temperature change of fingers of the patient's right arm on a surgical table during premedication and initial narcosis. Checkpoints $A, B$ and $C$ are situated in and around the nail bed of the fifth, fourth and third fingers, point $D$ and $E$ - on the phalanges of the second and first fingers, respectively. 


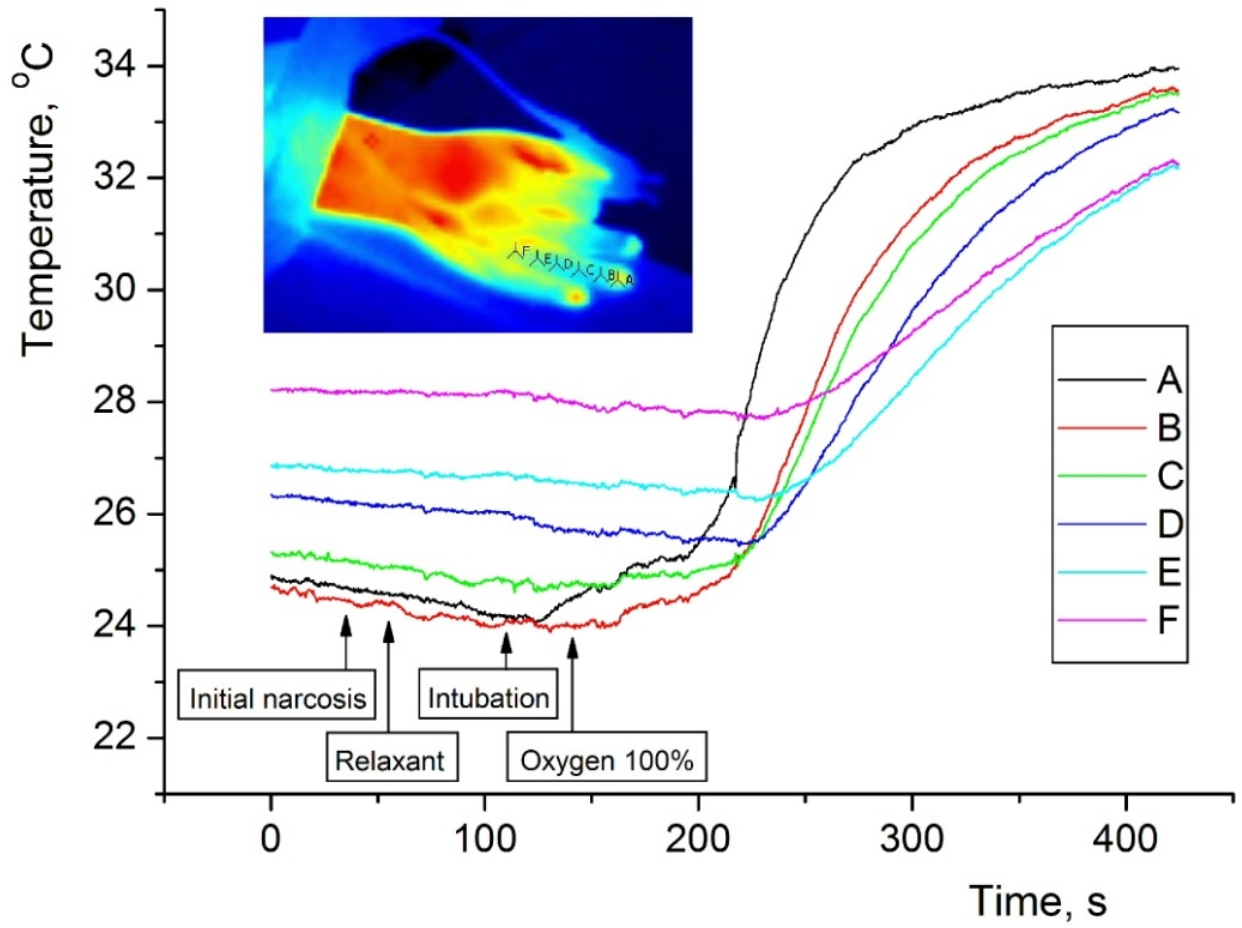

Fig. 7. Time profiles of the temperature measured in several points along the patient's fourth finger on the stage of premedication and initial narcosis before surgical operation. Checkpoints $A-F$ are located proximally starting from the nail bed. 